clearance of amyloid, with increased risk of developing Alzheimer's disease (8). Other neurodegenerative diseases also result from aberrant protein accumulation in the interstitial space of the brain. Prion diseases, such as Creutzfeld-Jacob disease, Parkinson's disease, and frontotemporal dementia, may result from cell-to-cell transmission of protein in the extracellular, interstitial space (9). Change in the kinetics of clearance of such proteins may be important in determining the rate of progression of these neurodegenerative disorders. Furthermore, the concentration and the distribution of molecules in the interstitial space of the brain are critical not only in chronic neurodegenerative disorders. Accumulation of interstitial fluid following mechanical and metabolic insults produces brain edema and neurologic symptoms, including coma and death. Using contrast-enhanced MRI to assess the kinetics of paravascular CSF flow may give important information about the impending appearance, and clinical progression, of this clinically important complication.

Address correspondence to: Warren J. Strittmatter, Duke University Medical Center, Bryan Research Bldg., Room 227, Durham, North Carolina 27710, USA. Phone: 919.684.0053; Fax: 919.681.7198; E-mail: warren@neuro.duke.edu.

1. Hajdu SI. A note from history: Discovery of the cerebrospinal fluid. Annals of Clin and Lab Sci. 2003;33(3):334-336.

2. Boulton M, et al. Drainage of CSF through lymphatic pathways and arachnoid villi in sheep: Mea- surement of ${ }^{125} \mathrm{I}$-albumin clearance. Neuropathol Appl Neurobiol. 1996;22(4):325-333.

3. Johnston M, et al. Evidence of connections between cerebrospinal fluid and nasal lymphatic vessels in humans, non-human primates and other mammalian species. Cerebrospinal Fluid Res. 2004;1(1):2.

4. Sakka L, Coll G, Chazal J. Anatomy and physiology of cerebrospinal fluid. Eur Ann Otorbinolaryngol Head Neck Dis. 2011;128(6):309-316.

5. Iliff JJ, et al. A paravascular pathway facilitates CSF flow through the brain parenchyma and the clearance of interstitial solutes, including amyloid b. Sci Transl Med. 2012;4(147):147ra111.

6. Iliff JJ, et al. Brain-wide pathway for waste clearance captured by contrast-enhanced MRI. J Clin Invest. 2013;123(3):1299-1309.

7. Huang Y, Mucke L. Alzheimer mechanisms and therapeutic strategies. Cell. 2012;148(6):1204-1222.

8. Jonsson T, et al. Variant of TREM2 associated with the risk of Alzheimer's disease. N Engl J Med. 2013; 368(2):107-116.

9. Frost B, Jacks RL, Diamond MI. Propagation of tau misfolding from the outside to the inside of a cell. J Biol Chem. 2009;284(19):12845-12852.

\title{
Calcium flux and endothelial dysfunction during acute lung injury: a STIMulating target for therapy
}

\author{
Eric J. Seeley, ${ }^{1}$ Paul Rosenberg, ${ }^{2}$ and Michael A. Matthay ${ }^{1}$
}

\begin{abstract}
${ }^{1}$ Cardiovascular Research Institute and Departments of Medicine and Anesthesia, UCSF, San Francisco, California, USA. ${ }^{2}$ Department of Medicine,
\end{abstract} Duke University, Durham, North Carolina, USA.

\begin{abstract}
Bacterial pathogen-associated molecular pattern molecules (PAMPs) such as LPS activate the endothelium and can lead to lung injury, but the signaling pathways mediating endothelial injury remain incompletely understood. In a recent issue of the JCI, Gandhirajan et al. identify STIM1, an ER calcium sensor, as a key link between LPS-induced ROS, calcium oscillations, and endothelial cell (EC) dysfunction. In addition, they report that BTP2, an inhibitor of calcium channels, attenuates lung injury. This study identifies a novel endothelial signaling pathway that could be a future target for the treatment of lung injury.
\end{abstract}

During infection, circulating bacterial products and endogenous cytokines stimulate the endothelium, setting off a cascade of vascular activation, including the increased expression of vascular adhesion molecules and regional increases in endothelial permeability. This response, the result of millennia of warfare between mammals and microbes, is beneficial during compartmentalized infections such as those of soft tissue and pneumonia. The alterations in vascular adhesion and permeability enable neutrophils and

Conflict of interest: The authors have declared that no conflict of interest exists.

Citation for this article: J Clin Invest. doi:10.1172/ JCI68093. monocytes to penetrate infected tissues, where they sequester and kill pathogens. However, similar physiological responses in vulnerable vascular beds, such as the capillary networks of the lungs and kidneys, can lead to multiple organ failure in critically ill patients. Achieving a deeper understanding of the signals that regulate vascular integrity during host defense and organ injury would be an important step in reducing vascular injury during human sepsis.

\section{The endothelium during sepsis and lung injury}

A major focus of recent research is the function of TLR4 within the vascular endothelium, where, through an inter- action with LPS, it plays a critical role as both protector and protagonist during sepsis and multiple organ failure. Mice that express TLR4 exclusively on ECs can detect and clear intraperitoneal E. coli infection as rapidly as wild-type counterparts (1). Thus, the endothelium, without the help of TLR4-expressing immune cells, can sense and eradicate intravascular infection. In addition to its role in immune surveillance and activation, the signaling pathways downstream of endothelial TLR4 are critical to the pathogenesis of organ injury, as illustrated by a mouse model in which a degradation-resistant form of $\mathrm{I} \mathrm{B}$, the cytoplasmic inhibitor of NF- $\kappa \mathrm{B}$, is expressed in ECs (2). These mice maintain the ability to sense and clear pathogens, yet have decreased organ injury and improved survival during LPS- or E. coli-induced peritonitis $(2,3)$. Thus, the endothelium is both a sensor of infection and a mediator of septic organ injury. Understanding how perturbations in TLR4 signaling lead to endothelial dysfunction would be a major step toward treating vascular dysfunction during sepsis. 
A
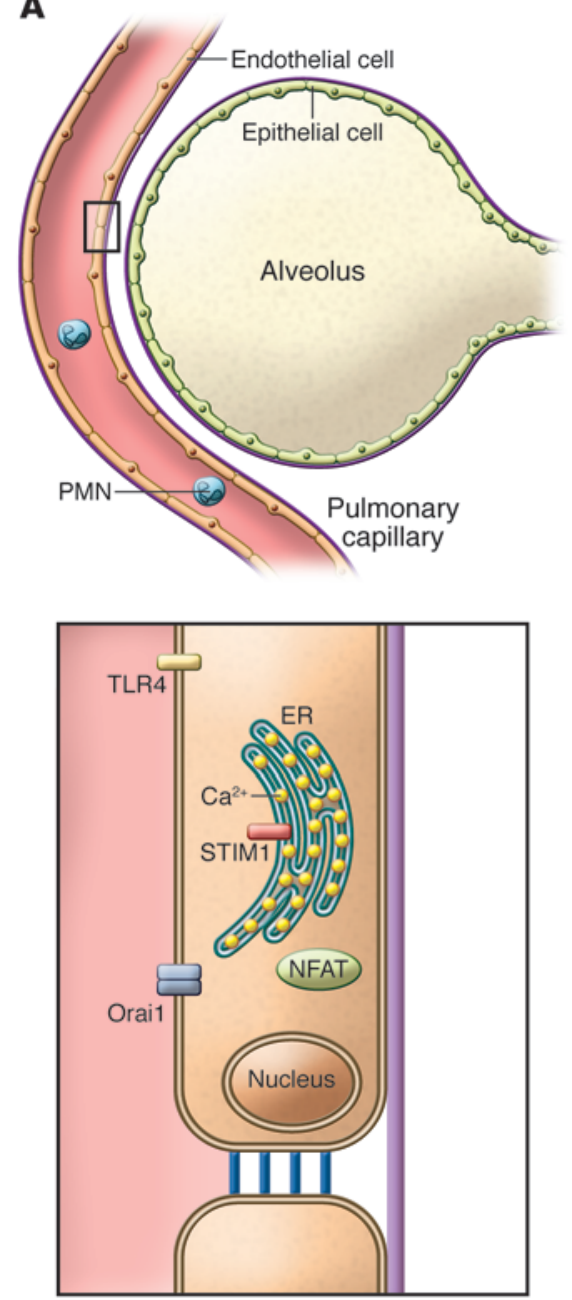

Normal pulmonary endothelium

Normal gas exchange

Stable alveolar-capillary barrier
B
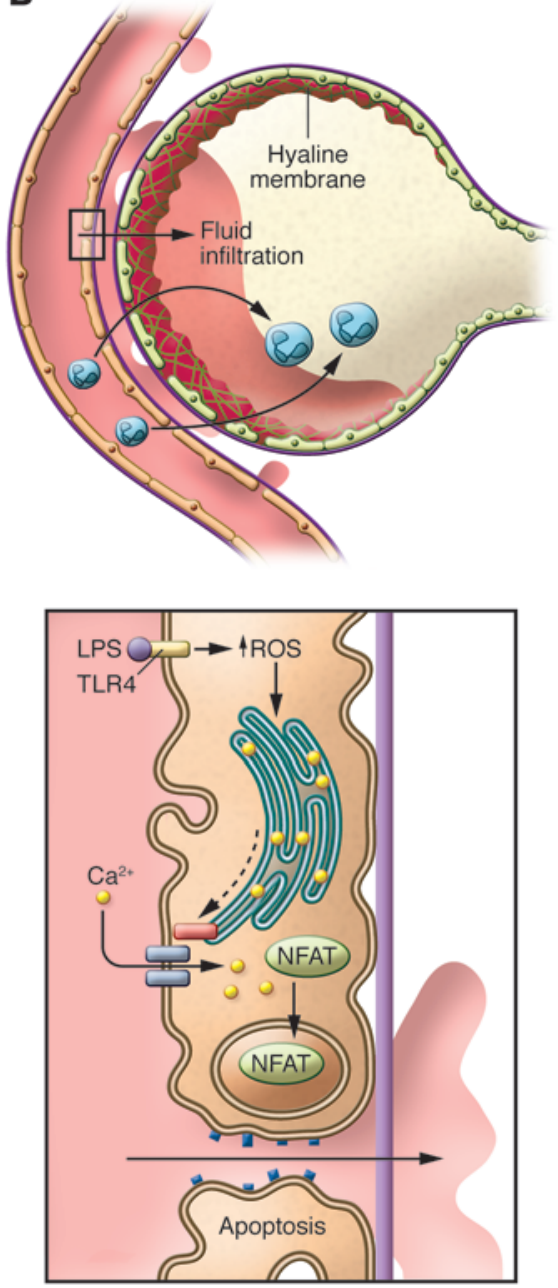

LPS-induced alterations Increased ROS

Increased $\mathrm{Ca}^{2+}$ oscillations

Endothelial cell apoptosis

Breakdown of alveolar-capillary barrier

Impaired gas exchange

\section{C}
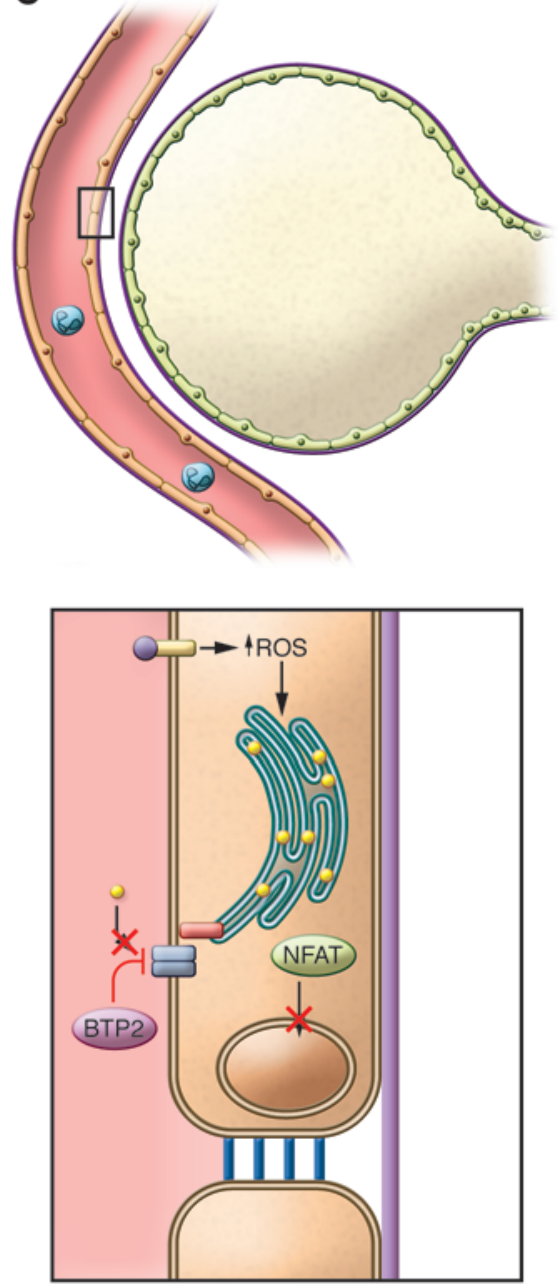

BTP2 inhibits LPS-induced endothelial injury

Increased ROS

$\mathrm{Ca}^{2+}$ oscillations inhibited

No endothelial apoptosis

Maintained alveolar-capillary barrier

Normal gas exchange

\section{Figure 1}

STIM1-mediated calcium oscillations connect TLR4 signals and endothelial dysfunction during lung injury. (A) The normal pulmonary endothelium maintains a tight barrier between endothelial cells, the interstitium, and the alveolar space. This preserves alveolar gas exchange. (B) LPSstimulated endothelial activation leads to ROS accumulation, calcium oscillations, and NFAT-mediated loss of barrier function. This allows leakage of protein-rich edema fluid into the alveolus, causing impaired gas exchange and hypoxemia. (C) BTP2 therapy inhibits calcium flux through Orai1 channels, dissociates ROS from NFAT nuclear translocation, inhibits subsequent endothelial injury and apoptosis.

Calcium flux connects TLR4 to NFATmediated endothelial dysfunction

Alterations in the frequency and amplitude of cytosolic $\mathrm{Ca}^{2+}$ levels have long been recognized as critical mediators of cell contraction and gene expression (4). Intriguingly, LPS bound to TLR4 can induce high-frequency $\mathrm{Ca}^{2+}$ oscillations and therefore alter EC permeability and gene expression profiles associated with acute lung injury (ALI). Mechanistically, $\mathrm{Ca}^{2+}$ oscillations involve both $\mathrm{Ca}^{2+}$ release from internal stores (from the IP3R) and $\mathrm{Ca}^{2+}$ entry via channels located in the cell membrane (e.g., TRPC6 and SOCE channels). In a recent issue of the JCI, Gandhirajan et al. uncovered the importance of store-operated $\mathrm{Ca}^{2+}$ entry (SOCE) in TLR4mediated EC dysfunction during ALI (5). SOCE involves the integrated actions of stromal interacting molecule 1 (STIM1), the $\mathrm{Ca}^{2+}$ sensor located in the ER membrane, and Orai1, the SOCE channel located in the plasma membrane. Depletion of internal stores reduces the amount of $\mathrm{Ca}^{2+}$ bound to EF hand motifs of the ER luminal domain of STIM1. Next, STIM1 oligomerizes and migrates from the internal ER to sites adjacent the plasma membrane (6) where Orai1 is activated, and $\mathrm{Ca}^{2+}$ enters the cell to refill stores and perpetuate $\mathrm{Ca}^{2+}$ oscillations. The importance of SOCE signaling is highlighted by the identification of patients harboring mutations in both STIM1 and ORAI1. These patients manifest a complex clinical syndrome involving 
immunodeficiency, hepatosplenomegaly, autoimmune hemolytic anemia, thrombocytopenia, muscular hypotonia, and defective enamel dentition $(7,8)$. The syndrome is invariably lethal, as patients succumb to overwhelming sepsis.

Mouse models that recapitulate this syndrome have been described, but only by achieving tissue-specific deletion of STIM1 or Orai1 in mice is it possible to investigate the physiologic role of SOCE. Gandhirajan et al. used endothelial cellspecific calcium sensor-knockout mice (Stim $1^{\triangle E C}$ mice) in a model of indirect lung injury. In contrast to littermate controls, Stim $1^{\triangle E C}$ mice did not display quantitative evidence of lung injury after LPS treatment. Strikingly, the levels of circulating inflammatory cytokines after LPS injection were identical in Stim $1^{\triangle E C}$ mice and controls, suggesting that it was the alteration of endothelial signaling events in the pulmonary capillary bed of the mutant mice that prevented the loss of lung endothelial integrity. Furthermore, the major difference between Stim $1^{\triangle E C}$ and wild-type endothelial cells was the absence of $\mathrm{Ca}^{2+}$ oscillations in the mutants (5).

The authors found that LPS/TLR4 stimulation influenced NFAT, a Rel A family transcription factor (5), explaining in part how deletion of STIM1 might influence gene expression. It has been established previously (6) that STIM1-mediated SOCE provides the sustained $\mathrm{Ca}^{2+}$ entry necessary to maintain calcineurin-dependent activation of NFAT during immune cell activation and development in many cell types. In addition, TLR4 had been previously shown to activate NOTCH1 gene expression in valvular endothelial cells (9). However, a link between TLR4, STIM1, and NFAT in pulmonary vascular injury had not been established. This finding opens many new avenues for investigation. For example, what are the NFAT transcriptional targets that regulate vascular permeability, and might they include the adhesion molecule ICAM-1, as was suggested recently (10)? Do STIM1-mediated $\mathrm{Ca}^{2+}$ oscillations influence NF- $\kappa B$ signaling as well? Do NFAT and $\mathrm{NF}-\kappa \mathrm{B}$ cooperate synergistically to influence gene expression? Finally, the authors report that STIM1 mediates LPS-induced pulmonary endothelial cell apoptosis (5), which may be a critical component of the pathophysiology of human acute respiratory distress syndrome (ARDS) (11).

This work also sheds light on an emerging concept in the SOCE field. STIM1 oligomerization and migration can be modulated by various cellular factors, including CAMP, temperature, and ROS. In this way, STIM1 functions as a relay station for the cross talk among these different signal transduction pathways. Prior studies by this group indicated that oxidant stress can induce STIM1-mediated $\mathrm{Ca}^{2+}$ entry via plasma membrane-bound Orai channels (12). Oxidant stress can induce $S$-glutathionylation of cysteine residues within the luminal domain of STIM1. By sensing the oxidant stress, STIM1 acts as a coincidence detector to activate SOCE and alter mitochondrial oxidative metabolism. In the present work, the authors extend these findings by identifying TLR4 and NADPH oxidase (NOX2) as key components in this pathway (5). LPS/TLR4 signaling can activate NOX2 and thus elevate oxidant stress through increased ROS production. According to this model, STIM1 can sense not only the depletion of $\mathrm{Ca}^{2+}$ stores, but also changes in NOX2derived ROS through $S$-glutathionylation of cysteine residues.

\section{Calcium flux: a new target for therapy}

In addition to outlining the critical role of STIM1 in mediating calcium oscillations and vascular permeability after LPS injury, Gandhirajan et al. tested a therapy that uncouples TLR4-mediated ROS from Ora1 channel calcium flux by blocking Oria1 channels (5). The authors utilized BTP2, a pyrazole-derived inhibitor of calcium release-activated calcium (CRAC) channels, which was originally described as a $\mathrm{T}$ cell immunosuppressant (13). BTP2 was delivered 2 hours after systemic LPS administration and resulted in a striking reduction in endothelial cell calcium flux and a sharp decrease in measures of lung injury. A key difference between treatment with BTP2 and other immunosuppressants is that BTP2 acts downstream of TLR4induced ROS. Thus, the potential antimicrobial benefit of ROS is preserved during BTP2 treatment and is divorced from the endothelial injury and apoptosis that lead to organ injury (Figure 1).

Although these preclinical studies utilizing BTP2 in a mouse model of indirect lung injury appear promising, several important steps will be needed before BTP2 could be used to treat human lung injury. First, off-target effects, especially the effect of BTP2 on T cells, must be assessed. Second, BTP2-mediated inhibition of pulmonary endothelial dysfunction should be done in the presence of live bacterial infection to be certain that innate or adaptive immune responses to infection are not impaired. Third, experiments in a larger animal model will be needed before this therapy is ready for clinical trials. Finally, it will be critical to determine the optimal timing for BTP2 delivery. Could therapy be administered to septic patients before the development of organ injury, and could it improve endothelial function after the onset of organ dysfunction?

This study adds to the body of research that has identified endothelial dysfunction as a key lesion in animal models of infection and human sepsis and lung injury $(14,15)$. Others have demonstrated that endothelial barrier function can be enhanced through multiple approaches, including strengthening endothelial junctions, reinforcing the endothelial cytoskeleton, and modulating endothelial cell activation (16). Recent studies have targeted all three of these, with impressive results. London et al. showed that endothelial barriers can be tightened with a fragment of Slit2, which is an endogenous inhibitor of VEGF signaling. Delivery of a Slit2 fragment to human endothelial cells in vitro or to mice during infection decreased endothelial permeability and improved survival (17). Similarly, molecules that target the angiopoietin-1 (Ang-1)/Tie-2 axis restored vascular permeability to a more normal state and improved blood flow to skeletal muscle by inhibiting phosphorylation-mediated VE-cadherin degradation (18). Gandhirajan et al. add a new dimension to these studies by identifying calcium-mediated NFAT signaling as a potential pathway for pathologic endothelial activation.

Collectively, these studies provide a compelling rationale for human therapies that target the injured endothelium during the early phase of sepsis. Because clinical investigators are now more focused on identifying patients earlier in the course of sepsis and lung injury in the emergency department prior to admission to the intensive care unit (19), this approach may be feasible in the clinical setting of human sepsis.

Address correspondence to: Michael A. Matthay, University of California, 505 Parnassus Avenue, Moffitt Hospital, M-917, San Francisco, California 941430624, USA. Phone: 415.353.1206; Fax: 415.353.1990; E-mail: michael.matthay@ ucsf.edu. 
1. Andonegui G, et al. Mice that exclusively express TLR4 on endothelial cells can efficiently clear a lethal systemic Gram-negative bacterial infection. J Clin Invest. 2009;119(7):1921-1930.

2. Ye X, Ding J, Zhou X, Chen G, Liu SF. Divergent roles of endothelial NF-kappaB in multiple organ injury and bacterial clearance in mouse models of sepsis. J Exp Med. 2008;205(6):1303-1315.

3. Xu H, Ye X, Steinberg H, Liu SF. Selective blockade of endothelial NF-kappaB pathway differentially affects systemic inflammation and multiple organ dysfunction and injury in septic mice. J Pathol. 2009;220(4):490-498.

4. Berridge MJ. Calcium signalling remodelling and disease. Biochem Soc Trans. 2012;40(2):297-309.

5. Gandhirajan RK, et al. Blockade of NOX2 and STIM1 signaling limits lipopolysaccharide-induced vascular inflammation. J Clin Invest. 2013;123(2):887-902.

6. Soboloff J, Rothberg BS, Madesh M, Gill DL. STIM proteins: dynamic calcium signal transducers. Nat Rev Mol Cell Biol. 2012;13(9):549-565.

7. Feske $S$, et al. A mutation in Orail causes immune deficiency by abrogating CRAC channel function. Nature. 2006;441(7090):179-185.

8. Picard C, et al. STIM1 mutation associated with a syndrome of immunodeficiency and autoimmunity. N Engl J Med. 2009;360(19):1971-1980.

9. Zeng Q. Cross-talk between the Toll-like receptor 4 and Notch 1 pathways augments the inflammatory respons in the interstitital cells of stenotic human aortic values. Circulation. 2012; 126(11 Suppl 1):S222-S230.

10. Xue J, et al. NF-kappaB regulates thrombin-induced ICAM-1 gene expression in cooperaton with NFAT by binding to the intronic NF-kappaB site in the ICAM-1 gene. Physiol Genomics. 2009;38(1):42-53.

11. Ware LB, Matthay MA. The acute respiratory distress syndrome. N Engl J Med. 2000;342(18):1334-1349.

12. Madesh M, et al. Selective role for superoxide in Ins $\mathrm{P} 3$ receptor-mediated mitochondrial dysfunction and endothelial apoptosis. J Cell Biol. 2005; 170(7):1079-1090.

13. Zitt $\mathrm{C}$, et al. Potent inhibition of $\mathrm{Ca}^{2+}$ release-activated $\mathrm{Ca}^{2+}$ channels and T-lymphocyte activation by the pyrazole derivative BTP2. J Biol Chem. 2004; 279(13):12427-12437.

14. Rubin DB, et al. Elevated von Willebrand factor antigen is an early plasma predictor of acute lung injury in nonpulmonary sepsis syndrome. J Clin Invest. 1990;86(2):474-480.

15. Rowlands DJ, et al. Activation of TNFR1 ectodomain shedding by mitochondrial $\mathrm{Ca}^{2+}$ determines the severity of inflammation in mouse lung microvessels. J Clin Invest. 2011;121(5):1986-1999.

16. Goldenberg NM, Steinberg BE, Slutsky AS, Lee WL. Broken barriers: a new take on sepsis pathogenesis. Sci Transl Med. 2011;3(88):88ps25.

17. London NR, et al. Targeting Robo4-dependent Slit signaling to survive the cytokine storm in sepsis and influenza. Sci Transl Med. 2010;2(23):23ra19.

18. Alfieri A, et al. Angiopoietin-1 variant reduces LPSinduced microvascular dysfunction in a murine model of sepsis. Crit Care. 2012;16(5):R182.

19. Matthay MA, Ware LB, Zimmerman GA. The acute respiratory distress syndrome. J Clin Invest. 2012; 122(8):2731-2740. 